\title{
Front Matter: Volume 9150
}

, "Front Matter: Volume 9150," Proc. SPIE 9150, Modeling, Systems Engineering, and Project Management for Astronomy VI, 915001 (20 August 2014); doi: 10.1117/12.2075747

Event: SPIE Astronomical Telescopes + Instrumentation, 2014, Montréal, Quebec, Canada 


\section{Modeling, Systems Engineering, and Project Management for Astronomy VI}

George Z. Angeli

Philippe Dierickx

Editors

22-24 June 2014

Montréal, Canada

Sponsored by

SPIE

Cooperating Organizations

American Astronomical Society (United States) - Australian Astronomical Observatory (Australia) - Association of Universities for Research in Astronomy (AURA) • Canadian Astronomical Society (CASCA) (Canada) • Canadian Space Agency (Canada) • European Astronomical Society (Switzerland) • European Southern Observatory (Germany) • National Radio Astronomy Observatory • Royal Astronomical Society (United Kingdom) • Science \& Technology Facilities Council (United Kingdom)

Published by

SPIE 
The papers included in this volume were part of the technical conference cited on the cover and title page. Papers were selected and subject to review by the editors and conference program committee. Some conference presentations may not be available for publication. The papers published in these proceedings reflect the work and thoughts of the authors and are published herein as submitted. The publisher is not responsible for the validity of the information or for any outcomes resulting from reliance thereon.

Please use the following format to cite material from this book:

Author(s), "Title of Paper," in Modeling, Systems Engineering, and Project Management for Astronomy VI, edited by George Z. Angeli, Philippe DierickX, Proceedings of SPIE Vol. 9150 (SPIE, Bellingham, WA, 2014) Article CID Number.

ISSN: 0277-786X

ISBN: 9780819496188

Published by

SPIE

P.O. Box 10, Bellingham, Washington 98227-0010 USA

Telephone +1 3606763290 (Pacific Time) · Fax +1 3606471445

SPIE.org

Copyright (@ 2014, Society of Photo-Optical Instrumentation Engineers.

Copying of material in this book for internal or personal use, or for the internal or personal use of specific clients, beyond the fair use provisions granted by the U.S. Copyright Law is authorized by SPIE subject to payment of copying fees. The Transactional Reporting Service base fee for this volume is $\$ 18.00$ per article (or portion thereof), which should be paid directly to the Copyright Clearance Center (CCC), 222 Rosewood Drive, Danvers, MA 01923. Payment may also be made electronically through CCC Online at copyright.com. Other copying for republication, resale, advertising or promotion, or any form of systematic or multiple reproduction of any material in this book is prohibited except with permission in writing from the publisher. The CCC fee code is 0277-786X/14/\$18.00.

Printed in the United States of America.

Publication of record for individual papers is online in the SPIE Digital Library.

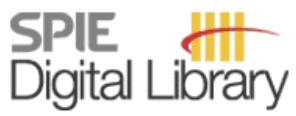

SPIEDigitalLibrary.org

Paper Numbering: Proceedings of SPIE follow an e-First publication model, with papers published first online and then in print and on CD-ROM. Papers are published as they are submitted and meet publication criteria. A unique, consistent, permanent citation identifier (CID) number is assigned to each article at the time of the first publication. Utilization of CIDs allows articles to be fully citable as soon as they are published online, and connects the same identifier to all online, print, and electronic versions of the publication. SPIE uses a six-digit CID article numbering system in which:

- The first four digits correspond to the SPIE volume number.

- The last two digits indicate publication order within the volume using a Base 36 numbering

system employing both numerals and letters. These two-number sets start with 00, 01, 02, 03, 04, $05,06,07,08,09,0 A, 0 B \ldots$. 0Z, followed by 10-1Z, 20-2Z, etc.

The CID Number appears on each page of the manuscript. The complete citation is used on the first page, and an abbreviated version on subsequent pages. Numbers in the index correspond to the last two digits of the six-digit CID Number. 


\title{
Contents
}

\author{
xiii Conference Committee \\ $\mathrm{xv}$ Introduction
}

\section{SESSION 1 PROJECT MANAGEMENT I}

915002 Success in large high-technology projects: What really works? [9150-1]

P. Crosby, Commonwealth Scientific and Industrial Research Organization (Australia)

915004 Generic documentation tree for science ground segments [9150-3]

F. Pérez-López, T. Lock, D. Texier, European Space Astronomy Ctr. (Spain)

915005 Tackling five main problem areas found in science (ground segment) project developments [9150-4]

T. Lock, F. Pérez-López, European Space Astronomy Ctr. (Spain)

\section{SESSION 2 PROJECT MANAGEMENT II}

915006 The tail wags the dog: managing large telescope construction projects with lagging requirements and creeping scope [9150-5]

M. Warner, National Solar Observatory (United States)

915007 Daniel K. Inouye Solar Telescope system safety [9150-6]

R. P. Hubbard, S. E. Bulau, S. Shimko, T. R. Williams, National Solar Observatory

(United States)

915008 Integrated Logistics Support approach: concept for the new big projects: E-ELT, SKA, CTA [9150-7]

G. Marchiori, F. Rampini, F. Formentin, European Industrial Engineering s.r.l. (Italy)

915009 De-mystifying earned value management for ground based astronomy projects, large and small [9150-8]

T. Norton, P. Brennan, M. Mueller, Harvard-Smithsonian Ctr. for Astrophysics (United States)

\section{SESSION 3 SYSTEM INTEGRATION, VERIFICATION, AND VALIDATION}

9150 OA The commissioning of Gaia (Invited Paper) [9150-9]

S. Els, European Space Astronomy Ctr. (Spain) and Astronomisches Rechen-Institut (Germany); T. Lock, G. Comoretto, European Space Astronomy Ctr. (Spain); G. Gracia, European Space Astronomy Ctr. (Spain) and Institute of Cosmos Sciences (Spain); W. O'Mullane, N. Cheek, European Space Astronomy Ctr. (Spain); A. Vallenari, European Space Astronomy Ctr. (Spain) and INAF - Osservatorio Astronomico di Padova (Italy) 
$9150 \mathrm{OB}$ The ALMA assembly, integration, and verification project: a retrospective analysis (Invited Paper) [9150-10]

B. Lopez, ALMA (Chile); L. B. G. Knee, National Research Council Canada (Canada); H. Jager, N. Whyborn, ALMA (Chile); J. McMullin, National Solar Observatory (United States); R. Murowinski, National Research Council Canada (Canada); A. Peck, National Radio Astronomy Observatory (United States); S. Corder, ALMA (Chile) and National Radio Astronomy Observatory (United States)

9150 OC Daniel K. Inouye Solar Telescope: integration testing and commissioning planning [9150-11] S. Craig, S. E. Bulau, K. Gonzales, E. Hansen, B. Goodrich, R. P. Hubbard, E. Johansson, C. Liang, R. A. Kneale, W. McBride, P. Sekulic, T. R. Williams, National Solar Observatory (United States)

9150 OD MUSE dream conclusion: the sky verdict [9150-12]

P. Caillier, Ctr. de Recherche Astrophysique de Lyon, CNRS, Univ. Claude-Bernard Lyon I (France); M. Accardo, European Southern Observatory (Germany); L. Adjali, Ctr. de Recherche Astrophysique de Lyon, CNRS, Univ. Claude-Bernard Lyon I (France); H. Anwand, Georg-August-Univ. Göttingen (Germany); R. Bacon, D. Boudon, L. Capoani, E. Daguisé, Ctr. de Recherche Astrophysique de Lyon, CNRS, Univ. Claude-Bernard Lyon I (France); M. Dupieux, Observatoire Midi-Pyrénées, CNRS, Univ. Paul Sabatier (France); C. Dupuy, European Southern Observatory (Germany); M. Francois, Ctr. de Recherche Astrophysique de Lyon, CNRS, Univ. Claude-Bernard Lyon I (France); A. Glindemann, D. Gojak, European Southern Observatory (Germany); F. Gonté, N. Haddad, European Southern Observatory (Chile); G. Hansali, Ctr. de Recherche Astrophysique de Lyon, CNRS, Univ. Claude-Bernard Lyon I (France); T. Hahn, Leibniz-Institut für Astrophysik Potsdam (Germany); A. Jarno, Ctr. de Recherche Astrophysique de Lyon, CNRS, Univ. ClaudeBernard Lyon I (France); A. Kelz, Leibniz-Institut für Astrophysik Połsdam (Germany); C. Koehler, Georg-August-Univ. Göttingen (Germany); J. Kosmalski, F. Laurent, Ctr. de Recherche Astrophysique de Lyon, CNRS, Univ. Claude-Bernard Lyon I (France); M. Larrieu, Observatoire Midi-Pyrénées, CNRS, Univ. Paul Sabatier (France); J.-L. Lizon, European Southern Observatory (Germany); M. Loupias, Ctr. de Recherche Astrophysique de Lyon, CNRS, Univ. Claude-Bernard Lyon I (France); A. Manescau, European Southern Observatory (Germany); J. E. Migniau, Ctr. de Recherche Astrophysique de Lyon, CNRS, Univ. Claude-Bernard Lyon I (France); C. Monstein, ETH Zürich (Switzerland); H. Nicklas, Georg-August-Univ. Göttingen (Germany); L. Parès, Observatoire Midi-Pyrénées, CNRS, Univ. Paul Sabatier (France); A. Pécontal-Rousset, L. Piqueras, Ctr. de Recherche Astrophysique de Lyon, CNRS, Univ. Claude-Bernard Lyon I (France); R. Reiss, European Southern Observatory (Germany); A. Remillieux, E. Renault, Ctr. de Recherche Astrophysique de Lyon, CNRS, Univ. Claude-Bernard Lyon I (France); G. Rupprecht, European Southern Observatory (Germany); O. Streicher, Leibniz-Institut für Astrophysik Potsdam (Germany); R. Stuik, Leiden Observatory, Leiden Univ. (Netherlands); H. Valentin, Observatoire Midi-Pyrénées, CNRS, Univ. Paul Sabatier (France); J. Vernet, European Southern Observatory (Germany); P. Weilbacher, Leibniz-Institut für Astrophysik Potsdam (Germany); G. Zins, Institut de Planétologie et d'Astrophysique de Grenoble, CNRS, Univ. Joseph Fourier (France)

9150 OE Planning and reality of the final verification and on-site assembly of KMOS for the VLT [9150-13]

P. Rees, G. H. Davidson, A. E. Fairley, M. Cirasuolo, UK Astronomy Technology Ctr. (United Kingdom) 
9150 OF Feed array metrology and correction layer for large antenna systems in ASIC mixed signal technology [9150-14]

F. Centureli, G. Scotti, P. Tommasino, A. Trifiletti, Univ. degli Studi di Roma La Sapienza

(Italy); F. Romano, Rheinmetall Italia S.p.A. (Italy); R. Cimmino, A. Saitto, NITEL (Italy)

$91500 \mathrm{G}$ Overview of the LSST active optics system [9150-15]

D. Neill, G. Angeli, C. Claver, E. Hileman, J. DeVries, J. Sebag, B. Xin, National Optical Astronomy Observatory (United States)

$9150 \mathrm{OH} \quad$ Real time wavefront control system for the Large Synoptic Survey Telescope (LSST) [9150-16] G. Z. Angeli, B. Xin, C. Claver, LSST Corp. (United States); D. MacMartin, California Institute of Technology (United States); D. Neill, LSST Corp. (United States); M. Britton, the Optical Sciences Co. (United States); J. Sebag, S. Chandrasekharan, LSST Corp. (United States)

9150 OI NEAT breadboard system analysis and performance models [9150-17] F. Hénault, A. Crouzier, F. Malbet, P. Kern, G. Martin, P. Feautrier, E. Staedler, S. Lafrasse, A. Delboulbé, Institut de Planétologie et d'Astrophysique de Grenoble, CNRS, Univ. Joseph Fourier (France); J.-M. Le Duigou, Ctr. National d'Études Spatiales (France); C. Cara, Lab. AIM, CNRS, Univ. Paris Diderot (France); A. Léger, Institut d'Astrophysique Spatiale, CNRS, Univ. Paris Sud (France)

$91500 \mathrm{~J}$ Relative performance of dispersive and non-dispersive far-infrared spectrometer instrument architectures [9150-18]

B. Sibthorpe, W. Jellema, SRON Netherlands Institute for Space Research (Netherlands)

9150 OK Running AIM: initial data treatment and $\mu$-arcsec level calibration procedures for Gaia within the astrometric verification unit [9150-19]

D. Busonero, M. G. Lattanzi, M. Gai, INAF - Osservatorio Astronomico di Torino (Italy);

E. Licata, EURIX (Italy); R. Messineo, ALTEC S.p.a. (Italy)

\section{SESSION $5 \quad$ MODEL BASED SYSTEMS ENGINEERING}

$9150 \mathrm{OL} \quad$ Model based systems engineering for astronomical projects (Invited Paper) [9150-20]

R. Karban, L. Andolfato, P. Bristow, G. Chiozzi, M. Esselborn, M. Schilling, C. Schmid,

H. Sommer, M. Zamparelli, European Southern Observatory (Germany)

9150 OM Systems engineering in the Large Synoptic Survey Telescope project: an application of model based systems engineering (Invited Paper) [9150-21]

C. F. Claver, B. M. Selvy, G. Angeli, LSST Corp. (United States); F. Delgado, Cerro Tololo InterAmerican Observatory (United States); G. Dubois-Felsmann, P. Hascall, SLAC National Accelerator Lab. (United States); P. Lotz, National Optical Astronomy Observatory (United States); S. Marshall, SLAC National Accelerator Lab. (United States); G. Schumacher, Cerro Tololo Inter-American Observatory (United States); J. Sebag, National Optical Astronomy Observatory (United States)

9150 ON Using SysML for verification and validation planning on the Large Synoptic Survey Telescope (LSST) [9150-22]

B. M. Selvy, C. Claver, G. Angeli, LSST Corp. (United States) 
9150 OP Transient aero-thermal simulations for TMT [9150-24]

K. Vogiatzis, Thirty Meter Telescope Observatory Corp. (United States)

$91500 Q \quad$ Unsteady wind loads for TMT: replacing parametric models with CFD [9150-25]

D. G. MacMartin, California Institute of Technology (United States) and Thirty Meter Telescope Observatory Corp. (United States); K. Vogiatzis, Thirty Meter Telescope Observatory Corp. (United States)

9150 OR Estimating dome seeing for LSST [9150-26]

J. Sebag, K. Vogiatzis, National Optical Astronomy Observatory (United States)

9150 OS Wavefront sensing and control performance modeling of the Thirty Meter Telescope for systematic trade analyses [9150-27]

C. Nissly, B.-J. Seo, M. Troy, Jet Propulsion Lab. (United States) and California Institute of Technology (United States); G. Chanan, Univ. of California, Irvine (United States); S. Roberts, J. Rogers, Thirty Meter Telescope Observatory Corp. (United States)

9150 OT TOAD: a numerical model for the 4MOST instrument [9150-28]

R. Winkler, D. M. Haynes, O. Bellido-Tirado, Leibniz-Institut für Astrophysik Potsdam (Germany); W. Xu, Optical System Engineering (Germany); R. Haynes, Leibniz-Institut für Astrophysik Potsdam (Germany)

\section{SESSION 7 SYSTEMS ENGINEERING I}

9150 OU Systems engineering plan for the construction phase of the E-ELT [9150-29]

J. C. Gonzalez, H. Kurlandczyk, D. Schneller, European Southern Observatory (Germany)

9150 OV Systems engineering of the Thirty Meter Telescope for the construction phase [9150-30] S. Roberts, J. Rogers, H. Thompson, K. Vogiatzis, D. MacMartin, E. Wilde, Thirty Meter Telescope Observatory Corp. (United States); M. Troy, B.-J. Seo, C. Nissly, Jet Propulsion Lab. (United States)

9150 OW The Paving Stones: initial feed-back on an attempt to apply the AGILE principles for the development of a CubeSat space mission to Mars [9150-31]

B. Segret, A. Semery, Lab. d'Etudes Spatiales et d'Instrumentation en Astrophysique, Observatoire de Paris (France); J. Vannitsen, National Cheng Kung Univ. (Taiwan);

B. Mosser, Lab. d'Etudes Spatiales et d'Instrumentation en Astrophysique, Observatoire de Paris (France); J.-J. Miau, J.-C. Juang, National Cheng Kung Univ. (Taiwan); F. Deleflie, Observatoire de Paris, CNRS, Univ. Pierre et Marie Curie (France)

9150 0X Automatic performance budget: towards a risk reduction [9150-32]

P. Laporte, GEPI, Observatoire de Paris, CNRS (France); S. Blake, J. Schmoll, Durham Univ. (United Kingdom); C. Rulten, LUTh, Observatoire de Paris, CNRS (France); D. Savoie, Institut d'Optique Graduate School (France) 
9150 OY Project management for complex ground-based instruments: MEGARA plan [9150-51] M. L. García-Vargas, A. Pérez-Calpena, FRACTAL S.L.N.E (Spain); A. Gil de Paz, J. Gallego, Univ. Complutense de Madrid (Spain); E. Carrasco, Instituto Nacional de Astrofísica, Óptica y Electrónica (Mexico); R. Cedazo, Univ. Politécnica de Madrid (Spain); J. Iglesias, Instituto de Astrofísica de Andalucía (Spain)

\section{SESSION $8 \quad$ SYSTEMS ENGINEERING II}

$91500 Z$ System modeling of the Thirty Meter Telescope alignment and phasing system [9150-36] F. G. Dekens, B.-J. Seo, M. Troy, Jet Propulsion Lab. (United States)

915010 Systems and context modeling approach to requirements analysis [9150-37] A. Ahuja, G. Muralikrishna, P. Patwari, S. C., N. Swaminathan, H. Vin, Tata Research Development and Design Ctr. (India)

915011 TMT telescope structure thermal model [9150-72]

K. Vogiatzis, A. Sadjadpour, S. Roberts, Thirty Meter Telescope Observatory Corp. (United States)

915012 Heat balance and thermal management of the TMT Observatory [9150-83] H. Thompson, K. Vogiatzis, Thirty Meter Telescope Observatory Corp. (United States)

915013 Polarimetric analysis of the Thirty Meter Telescope (TMT) for modeling instrumental polarization characteristics [9150-76]

J. Atwood, National Research Council Canada (Canada); W. Skidmore, Thirty Meter Telescope Observatory Corp. (United States); G. C. Anupama, R. M. Anche, Indian Institute of Astrophysics (India); K. Reddy, Aryabhatta Research Institute of Observational Sciences (India); A. Sen, Assam Univ. (India)

\section{SESSION 9 SYSTEM MODELING II}

915014 An end-to-end simulation framework for the Large Synoptic Survey Telescope [9150-38] A. J. Connolly, Univ. of Washington (United States); G. Z. Angeli, LSST Corp. (United States); S. Chandrasekharan, National Optical Astronomy Observatory (United States); C. F. Claver, K. Cook, LSST Corp. (United States); Z. Ivezic, R. L. Jones, K. S. Krughoff, Univ. of Washington (United States); E.-H. Peng, J. Peterson, Purdue Univ. (United States); C. Petry, LSST Corp. (United States); A. P. Rasmussen, SLAC National Accelerator Lab. (United States); S. T. Ridgway, A. Saha, National Optical Astronomy Observatory (United States); G. Sembroski, Purdue Univ. (United States); J. vanderPlas, P. Yoachim, Univ. of Washington (United States)

915015 The LSST operations simulator [9150-39]

F. Delgado, Cerro Tololo Inter-American Observatory (Chile); A. Saha, S. Chandrasekharan, National Optical Astronomy Observatory (United States); K. Cook, Eureka Scientific (United States); C. Petry, LSST Corp. (United States); S. Ridgway, National Optical Astronomy Observatory (United States) 
915016 DPAC operations simulation to take up the Gaia challenge [9150-40]

S. G. Els, European Space Astronomy Ctr. (Spain) and Astronomisches Rechen-Institut (Germany); T. Lock, G. Comoretto, European Space Astronomy Ctr. (Spain); G. Gracia, European Space Astronomy Ctr. (Spain) and Institut of Cosmos Sciences (Spain); W. O'Mullane, N. Cheek, European Space Astronomy Ctr. (Spain); A. Vallenari, European Space Astronomy Ctr. (Spain) and INAF - Osservatorio Astronomico di Padova (Italy); D. Ordoñez, M. Beck, Astronomical Observatory of the Univ. of Geneva (Switzerland)

915017 A framework for modeling the detailed optical response of thick, multiple segment, large format sensors for precision astronomy applications [9150-41]

A. Rasmussen, SLAC National Accelerator Lab. (United States); P. Antilogus, P. Astier, LPNHE/IN2P3, CNRS, Univ. Pierre et Marie Curie (France); C. Claver, National Optical Astronomy Observatory (United States); P. Doherty, Harvard Univ. (United States);

G. Dubois-Felsmann, K. Gilmore, S. Kahn, SLAC National Accelerator Lab. (United States);

I. Kotov, Brookhaven National Lab. (United States); R. Lupton, Princeton Univ.

(United States); P. O'Connor, A. Nomerotski, Brookhaven National Lab. (United States);

S. Ritz, Univ. of California, Santa Cruz (United States); C. Stubbs, Harvard Univ.

(United States)

915018 Have confidence in your coronagraph: statistical analysis of high-contrast coronagraph dynamics error budgets [9150-42]

S. B. Shaklan, L. Marchen, L. Peterson, M. B. Levine, Jet Propulsion Lab. (United States)

\section{SESSION 10 SYSTEMS ENGINEERING III}

915019 Daniel K. Inouye Solar Telescope systems engineering update [9150-43]

S. Craig, E. Hansen, R. P. Hubbard, R. Kneale, National Solar Observatory (United States)

$91501 \mathrm{~A}$ Managing the system validation of the DKIST enclosure [9150-44]

J. Ariño, C. Gómez, G. Murga, P. Ampuero, R. Campo, AEC IDOM (Spain)

$91501 \mathrm{~B}$ Systems engineering implementation in the conceptual design phase of 4MOST [9150-45] O. Bellido-Tirado, R. Haynes, R. S. de Jong, O. Schnurr, J. Walcher, R. Winkler, Leibniz-Institut für Astrophysik Potsdam (Germany)

9150 1C From space to specs: requirements for 4MOST [9150-46]

O. Schnurr, C. J. Walcher, C. Chiappini, A. D. Schwope, O. Bellido-Tirado, R. Haynes, Leibniz-Institut für Astrophysik Potsdam (Germany); S. Feltzing, Lund Observatory (Sweden); R. McMahon, Univ. of Cambridge (United Kingdom); R. S. de Jong, Leibniz-Institut für Astrophysik Potsdam (Germany); W. Ansorge, RAMS-CON Management Consultants (Germany)

9150 1D Complexity in the MATISSE cold optics: a risk or a tool? [9150-47]

N. Tromp, F. Bettonvil, G. Aitink-Kroes, T. Agócs, R. Navarro, ASTRON (Netherlands) 
9150 1E Novel technique for tracking manpower and work packages: a useful tool for the team and management [9150-48]

R. Gill, G. Gracia, European Space Astronomy Ctr. (Spain); R. H. Lupton, Princeton Univ. (United States); W. O'Mullane, European Space Astronomy Ctr. (Spain)

$91501 \mathrm{H}$ Space radiation parameters for EUI and the sun sensor of Solar Orbiter, ESIO, and JUDE instruments [9150-52]

L. Rossi, L. Jacques, J.-P. Halain, E. Renotte, T. Thibert, D. Grodent, Univ. de Liège (Belgium)

915011 GAME/ISAS development status [9150-53]

M. Gai, A. Riva, D. Busonero, A. Vecchiato, M. G. Lattanzi, INAF - Osservatorio Astronomico di Torino (Italy); D. Gallieni, P. Lazzarini, A.D.S. International S.r.l. (Italy); G. Guglieri, Politecnico di Torino (Italy); I. Musso, P. Navone, Advanced Logistics Technology Engineering Ctr. (Italy)

$91501 \mathrm{~J}$ Thermal design and performance of the REgolith x-ray imaging spectrometer (REXIS) instrument [9150-54]

K. D. Stout, R. A. Masterson, Massachusetts Institute of Technology (United States)

$91501 \mathrm{~L}$ Design of one large telescope direct drive control system based on TMS320F28xx [9150-56] X.-L. Song, D.-X. Wang, C. Zhang, Z.-C. Zhang, Nanjing Institute of Astronomical Optics \& Technology (China); L.-Y. Chen, Nanjing Institute of Astronomical Optics \& Technology (China) and Univ. of Chinese Academy of Sciences (China); C.-Z. Ren, Nanjing Institute of Astronomical Optics \& Technology (China

9150 IN BIRDY: an interplanetary CubeSat to collect radiation data on the way to Mars and back to prepare the future manned missions [9150-58]

B. Segret, Lab. d'Etudes Spatiales et d'Instrumentation en Astrophysique, Observatoire de Paris (France); J. Vannitsen, M. Agnan, National Cheng Kung Univ. (Taiwan); A. Porquet, Observatoire de Paris, CNRS, and Univ. Pierre et Marie Curie (France); O. Sleimi, National Cheng Kung Univ. (Taiwan); F. Deleflie, Observatoire de Paris, CNRS, and Univ. Pierre et Marie Curie (France); J.-J. Miau, J.-C. Juang, K. Wang, National Cheng Kung Univ. (Taiwan)

915010 Chilean virtual observatory and integration with ALMA [9150-59]

M. Solar, W. Fariña, Univ. Técnica Federico Santa María (Chile); D. Mardones, Univ. de Chile (Chile); J. Antognini, Univ. Técnica Federico Santa María (Chile); K. Pichara, Pontificia Univ. Católica de Chile (Chile); N. Nagar, Univ. de Concepción (Chile); V. Parada, Univ. de Santiago de Chile (Chile); J. Ibsen, L. Nyman, ALMA (Chile); J. Marroquin, Univ. Técnica Federico Santa María (Chile)

$91501 Q \quad$ Plate coil thermal test bench for the Daniel K. Inouye Solar Telescope (DKIST) carousel cooling system [9150-62]

L. Phelps, National Solar Observatory (United States); G. Murga, AEC IDOM (United States);

G. Montijo Jr., D. Hauth, National Solar Observatory (United States) 
$91501 R \quad$ FRIDA diffraction limited NIR instrument: the challenges of its verification processes [9150-63]

B. Sánchez, C. Keiman, C. Espejo, S. Cuevas, L. C. Álvarez, O. Chapa, R. Flores-Meza, J. Fuentes, L. Garcés, G. Lara, J. López, R. Rodríguez, A. Watson, Univ. Nacional Autónoma de México (Mexico); V. Bringas, A. Corrales, D. Lucero, A. Rodríguez, B. Rodríguez, D. Torres, J. Uribe, Ctr. de Ingenieria y Desarrollo Industrial (Mexico)

9150 1S DKIST enclosure modeling and verification during factory assembly and testing [9150-64] I. Larrakoetxea, AEC IDOM (United States); W. McBride, H. K. Marshall, National Solar Observatory (United States); G. Murga, AEC IDOM (United States)

$91501 \mathrm{~T}$ Structural influences on intensity correlation interferometry [9150-65] M. Harris, A. Maji, The Univ. of New Mexico (United States)

$91501 \mathrm{U} \quad$ System model of an image stabilization system [9150-66]

M. Carmona, J. M. Gómez, D. Roma, A. Casas, M. López, J. Bosch, A. Herms, J. Sabater, Univ. de Barcelona (Spain); R. Volkmer, F. Heidecke, T. Mave, E. Nakai, W. Schmidt, Kiepenheuer-Institut für Sonnenphysik (Germany)

$91501 \mathrm{~W}$ Cosmic non-TEM radiation and synthetic feed array sensor system in ASIC mixed signal technology [9150-69]

F. Centureli, G. Scotti, P. Tommasino, A. Trifiletti, Univ. degli Studi di Roma La Sapienza (Italy); F. Romano, Rheinmetall Italia S.p.A. (Italy); R. Cimmino, A. Saitto, NITEL (Italy)

$91501 \mathrm{X} \quad$ The OTP-model applied to the Aklim site database [9150-70]

K. Mraini, A. Jabiri, Z. Benkhaldoun, A. Bounhir, Y. Hach, M. Sabil, A. Habib, Univ. Cadi Ayyad (Morocco)

$91501 Y \quad$ One of align metrologies for Antarctic telescopes [9150-71]

Z. Li, Nanjing Institute of Astronomical Optics \& Technology (China) and Graduate Univ. of Chinese Academy of Sciences (China); X. Yuan, Nanjing Institute of Astronomical Optics \& Technology (China) and Chinese Ctr. for Antarctic Astronomy (China)

$91501 \mathrm{Z}$ The Basic Angle Monitoring (BAM) software tool in the context of Gaia's astrometric verification [9150-73]

A. Riva, M. G. Lattanzi, R. Drimmel, M. Gai, D. Busonero, R. Buzzi, INAF - Osservatorio Astrofisico di Torino (Italy); M. Pecoraro, EURIX (Italy); F. Russo, INAF - Osservatorio Astrofisico di Torino (Italy); R. Messineo, ALTEC S.p.a. (Italy)

915020 Error reduction and modeling for hexapod positioners of secondary mirrors for large ground-based telescopes [9150-74]

R. C. Sneed, P. J. Keas, Moog CSA Engineering (United States)

$915021 \quad$ Integrated modeling for parametric evaluation of smart x-ray optics [9150-75]

S. Dell'Agostino, M. Riva, D. Spiga, S. Basso, M. Civitani, INAF - Osservatorio Astronomico di Brera (Italy)

915022 Wind responses of Giant Magellan telescope [9150-77]

B. Irarrazaval, GMTO Corp. (United States); C. Buleri, Quartus Engineering Inc.

(United States); M. Johns, GMTO Corp. (United States) 
915023 Target allocation yields for massively multiplexed spectroscopic surveys with fibers [9150-78]

W. Saunders, S. Smedley, P. Gillingham, Australian Astronomical Observatory (Australia);

J. E. Forero-Romero, Univ. de los Andes (Colombia); S. Jouvel, Institut de Ciències de l'Espai (Spain); B. Nord, Fermi National Accelerator Lab. (United States)

915024 The ASTRI SST-2M prototype for the next generation of Cherenkov telescopes: a single framework approach from requirement analysis to integration and verification strategy definition [9150-79]

M. Fiorini, N. La Palombara, L. Stringhetti, INAF - IASF Milano (Italy); R. Canestrari, INAF Osservatorio Astronomico di Brera (Italy); O. Catalano, INAF - Istituto di Astrofisica Spaziale e Fisica Cosmica di Palermo (Italy); E. Giro, INAF - Osservatorio Astronomico di Padova (Italy); G. Leto, INAF - Osservatorio Astrofisico di Catania (Italy); M. C. Maccarone, INAF Istituto di Astrofisica Spaziale e Fisica Cosmica di Palermo (Italy); G. Pareschi, INAF Osservatorio Astronomico di Brera (Italy); G. Tosti, Univ. degli Studi di Perugia (Italy); S. Vercellone, INAF - Istituto di Astrofisica Spaziale e Fisica Cosmica di Palermo (Italy)

915025 E-ELT requirements management [9150-80]

D. Schneller, European Southern Observatory (Germany)

915026 System engineering at the MEGARA project [9150-81]

A. Pérez-Calpena, M. L. García-Vargas, FRACTAL S.L.N.E (Spain); A. Gil de Paz, J. Gallego Maestro, Univ. Complutense de Madrid (Spain); E. Carrasco Licea, Instituto Nacional de Astrofísica, Óptica y Electrónica (Mexico); F. Sánchez Moreno, Univ. Politécnica de Madrid (Spain); J. Iglesias-Páramo, Instituto de Astrofísica de Andalucía (Spain)

915027 Introducing questionnaire technique to interface with multi-instrument teams for science operations [9150-82]

F. Pérez-López, S. de la Fuente, European Space Astronomy Ctr. (Spain)

Author Index 
Proc. of SPIE Vol. $9150915001-12$

Downloaded From: https://www.spiedigitallibrary.org/conference-proceedings-of-spie on 26 Apr 2023 Terms of Use: https://www.spiedigitallibrary.org/terms-of-use 


\section{Conference Committee}

Symposium Chairs

Gillian S. Wright, UK Astronomy Technology Centre (United Kingdom) Luc Simard, National Research Council Canada (Canada)

Symposium Co-chairs

Colin Cunningham, UK Astronomy Technology Centre (United Kingdom)

Masanori lye, National Astronomical Observatory of Japan (Japan)

Conference Chairs

George Z. Angeli, LSST Corporation (United States)

Philippe Dierickx, European Southern Observatory (Germany)

Conference Program Committee

Simon C. Craig, National Solar Observatory (United States)

Sebastian G. Els, European Space Astronomy Centre (Spain)

Gary E. Mosier, NASA Goddard Space Flight Center (United States)

Scott Roberts, Thirty Meter Telescope Observatory Corporation

(Canada)

Hermine Schnetler, UK Astronomy Technology Centre

(United Kingdom)

Michael Sheehan, Giant Magellan Telescope Project (United States)

Mitchell Troy, Jet Propulsion Laboratory (United States)

\section{Session Chairs}

1 Project Management I

George Z. Angeli, LSST Corporation (United States)

2 Project Management II

Sebastian G. Els, European Space Astronomy Centre (Spain)

3 System Integration, Verification, and Validation

Scott Roberts, Thirty Meter Telescope Observatory Corporation

(United States)

$4 \quad$ System Designs and Architectures

Hermine Schnetler, UK Astronomy Technology Centre

(United Kingdom) 
5 Model Based Systems Engineering

Sebastian G. Els, European Space Astronomy Centre (Spain)

6 System Modeling I

George Z. Angeli, LSST Corporation (United States)

7 Systems Engineering I

Simon Craig, National Solar Observatory (United States)

Tuesday Plenary Session

Gillian S. Wright, UK Astronomy Technology Centre (United Kingdom)

8 Systems Engineering II

Michael Sheehan, Giant Magellan Telescope Project (United States)

9 System Modeling II

Mitchell Troy, Jet Propulsion Laboratory (United States)

10 Systems Engineering III

Philippe Dierickx, European Southern Observatory (Germany) 


\section{Introduction}

This conference marked the 10th anniversary of launching a gathering dedicated to Systems Modeling and Systems Engineering as they are applied on telescope and instrument developments in astronomy.

Until the end of the 20th century, telescopes and instruments were difficult to make, but conceptually simple systems. New technologies, new processes, new materials were needed to make them possible, and some of these undertakings would still be considered as mighty challenges today. However, these telescopes and instruments belonged to the machine world. In this world, it is the parts that matter most; understanding them is broadly sufficient to understand the system, and make it work. This seems no longer true. Instruments, and after them, the notionally simpler telescopes, became functionally more complex. They have been transiting into the systems world, whereby overall system properties can no longer be approximated by the sum of individual ones. In this world, engineers must cope with difficulty and complexity. The former is usually the domain of the specialist; the latter, of the systems engineer.

The evolution of this conference over the last decade reflects this transition. In Glasgow, the conference was dominated by modeling, indicating the prevalence of technical challenges on the minds of our colleagues. In the relatively short timeframe of 10 years, the tide has changed; not just the large number of systems engineering papers are demonstrating it, but also their high quality.

In 2004, the goal was set for the community, as well as for the conference: "Systems engineering has existed in space astronomy projects since the very first mission. With the ever increasing complexity and cost of ground-based facilities, it should also be at the core of all astronomy technology projects." If this year's conference is any indication, we are getting there ...

Projects have started picking from a vast body of knowledge, adopting and adapting principles and methods to their particular needs, objectives, or culture. Through these proceedings, we hope the reader may find ideas about and solutions to problems he or she has to cope with, and, perhaps, eventually decide to share with the community his or her experience at the next occurrence of this conference. Keep the body of knowledge breathing! 
Proc. of SPIE Vol. $9150915001-16$

Downloaded From: https://www.spiedigitallibrary.org/conference-proceedings-of-spie on 26 Apr 2023 Terms of Use: https://www.spiedigitallibrary.org/terms-of-use 